\title{
Dual-Energy Subtraction Mammography
}

\author{
Taroh Asaga, Chihiro Masuzawa, Akira Yoshida, and Hirofumi Matsuura
}

Dual-energy subtraction mammography was performed for breast examinations. To obtain a dualenergy subtraction image with a digital radiography unit, high- and low-energy images were obtained at an appropriate time interval under different $x$-ray exposure conditions. In about $50 \%$ of the patients with breast cancer included in this study, we obtained better diagnostic accuracy with dual-energy subtraction images than with conventional mammography. In some cases of breast cancer, it was possible to diagnose intraductal spread of this lesion on the subtracted images. Furthermore, abnormal lesions commonly observed on mammography in cases of fibrocystic disease tended to be erased on subtracted images. Thus, dual-energy subtraction mammography provided useful information for diagnosing breast diseases. However, there were several cases in which the subtracted images lacked sufficient image quality, and several technical problems with subtraction are thought to remain.

Copyright $\odot 1995$ by W.B. Saunders Company

KEY WORDS: dual-energy subtraction mammography, breast cancer, conventional mammography.

$\mathbf{M}$ AMMOGRAPHY is the examination of choice for diagnosis of breast diseases, and its diagnostic reliability is relatively high. However, on conventional mammograms obtained by the compression method (mammography), the normal soft tissue of the breast, tumors, calcium deposits, and other shadows sometimes overlap in such a way as to mask lesions or render the infiltration border unclear. Therefore, tumors might not be clearly differentiated from the adjacent mammary structure. In addition, sometimes overlapping mammary glands and soft tissue create so-called pseudotumorous lesions. Owing to these disadvantages of mammography, we clinically investigated the borders of cancer infiltration with digital dualenergy subtracted images. To examine the usefulness of this method, dual-energy subtraction mammography was performed with Fuji Com-

From the Department of Surgery and Radiology, Kanagawa Cancer Center Hospital, Yokohama, Japan.

Address reprint requests to Taroh Asaga, MD, Department of Surgery and Radiology, Kanagawa Cancer Center Hospital, 54-2, Nakao-cho, Asahi-ku, Yokohama 241, Japan.

Copyright $\odot 1995$ by W.B. Saunders Company 0897-1889/95/0801-1016\$3.00/0 puted Radiography (FCR) System (Fuji Medical Systems, Tokyo, Japan).

\section{DUAL-ENERGY SUBTRACTION METHOD}

A mammography was taken using a box-type exposure stand with a built-in moving grid of carbon fiber plates, against which the breast was pressed. Philips Mammo Diagnost UM (Philips, the Netherlands) was used for breast $\mathrm{x}$-ray equipment and different exposure energies (MO anode, $x$-ray tube, at 28 kilovolt (peak) and 40 $\mathrm{kVp}$ ) were used. We then recorded two breast $\mathrm{x}$-ray images on the imaging plates. Images on the two imaging plates were read by the FCR 7000 system. The position of each image was matched using a registration algorithm so that the data from the two images overlapped completely, and weighted subtraction processing was performed. We then read the image after the mammary glands were most conspicuously erased.

\section{RESULTS}

\section{Subtraction in Cancer Cases}

To investigate the usefulness of this subtraction method, we used this method in 42 cases of breast cancer that had been confirmed histopathologically. Mammography did not provide sufficient information in diagnosing these 42 cases of breast cancer. In 21 cases, subtracted images provided better information than mammography did. In 18 of 21 , better visualization of the tumor or border of the tumor could be obtained, and in the remaining 3, daughter nodules were visualized on subtracted images. In the other 20 cases, subtracted images were equivalent to conventional mammography (Table 1).

Table 1. Subtraction of Cancer Cases

Observation Better Than

With Mammography

Content of No. of Same as

Observations Cases Mammography

Tumor shadow or the border of

cancer infiltration is clearly

visualized 18

Daughter nodule is visualized 3

Total 21 

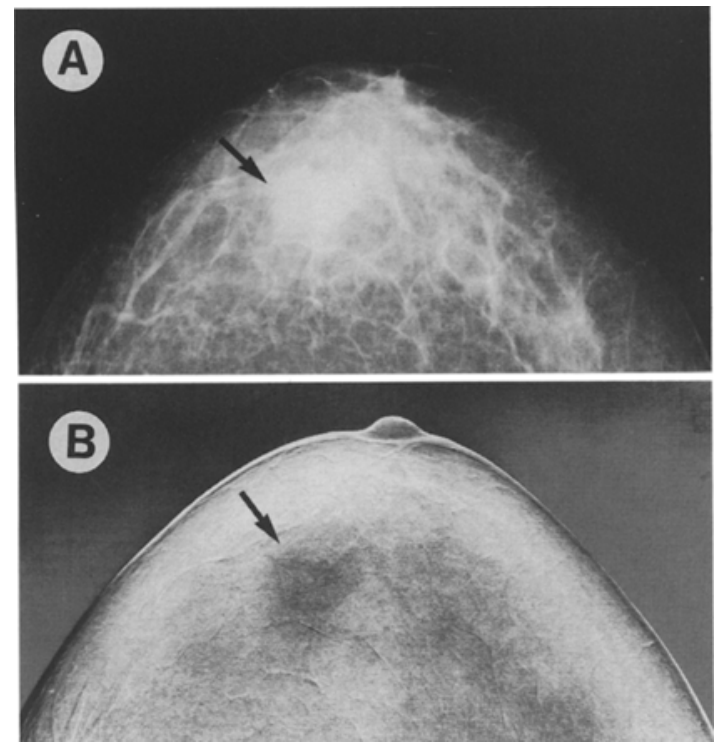

Fig 1. (A) Mammogram and (B) subtraction. The border of the tumor was clearly visualized in the subtracted image.
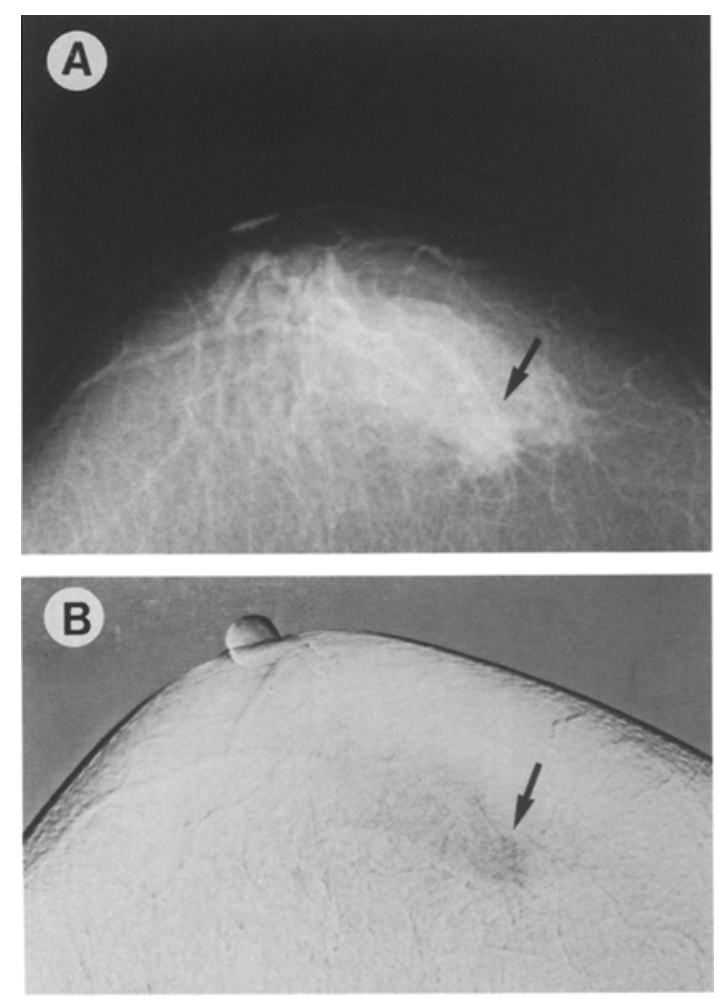

Fig 2. (A) Mammogram and (B) subtraction. Normal mammary glands were erased, resulting in clear visualization of the border of cancer infiltration.
In case 1 (Fig 1), a 58-year-old woman had a tumor detected in the subareolar portion, but the mammary glands overlapping the tumor prevented the tumor from being visualized clearly. With subtraction, the tumor could be clearly visualized. Histopathologically, this tumor was solid-tubular carcinoma.

In case 2 (Fig 2), a 48-year-old woman had breast cancer diagnosed from the spicular formation on mammography. However, the border of cancer infiltration was not clearly visualized because of the overlapping normal mammary gland. Thus, subtraction was performed to identify the border of the cancer. On the subtracted image, the normal mammary gland was erased, thus clarifying the border of cancer infiltration.

In case 3, a 45-year-old woman presented with a suspicious concentrated lesion on mammograms, but the tumor could not be clearly observed. The subtracted image clearly visualized two lesions with spicula.

\section{Subtraction in Benign Disease}

We examined subtracted images from cases that had tumor shadows, high-density areas,
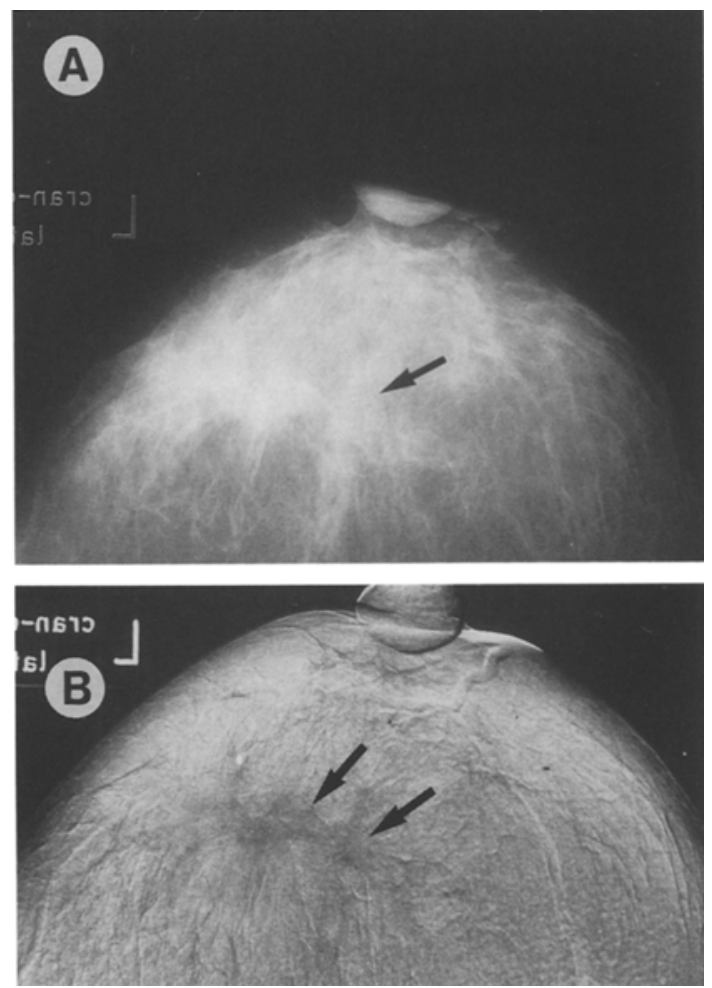

Fig 3. (A) Mammogram and (B) subtraction. With subtraction, the two tumors and spicular formation were visualized. 

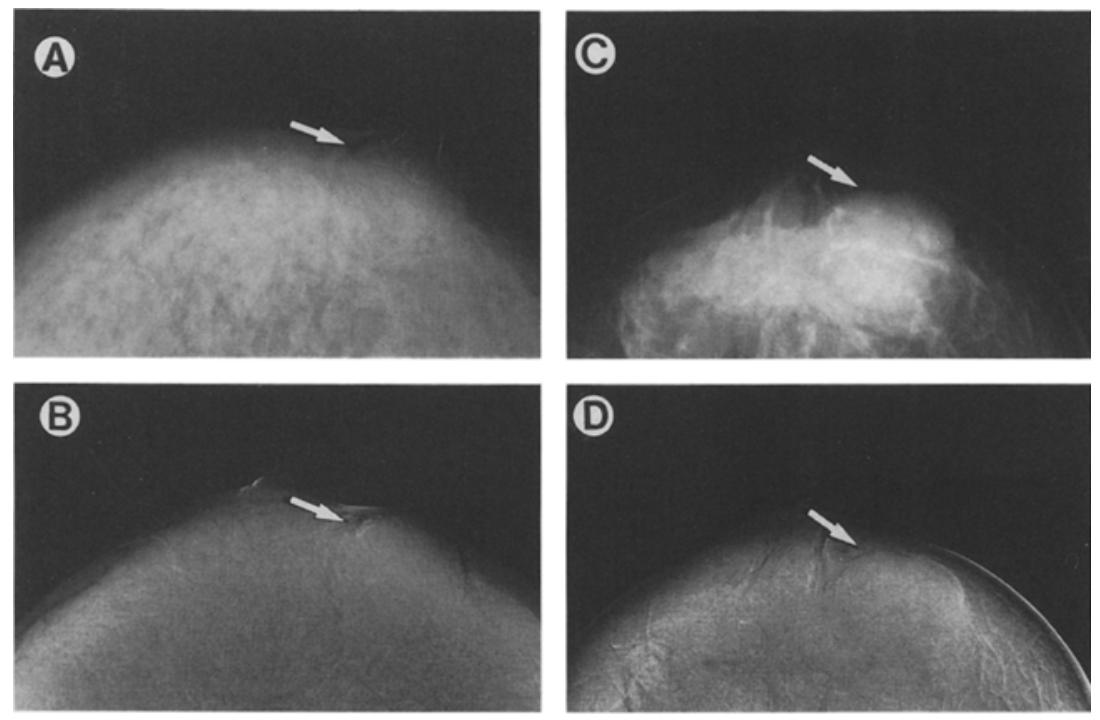

Fig 4. (A) Mammogram of cystic disease and (B) subtraction. The tumor has been erased. (C) Mammogram of fibroadenoma; (D) subtraction. Tumor has been erased.

and spicular formation on mammograms, but were diagnosed histopathologically as benign disease. In eight cases with fibrocystic disease and three with cysts showing tumor shadows, high-density areas, and spicula, the lesions were erased with subtracted images. The tumor shadow was also erased in two of the four cases of fibroadenoma, but in the remaining two cases, the tumor was not completely erased on the subtracted image. These results suggest that the benign lesions observed on mammography tend to be erased on subtracted images (Fig 4 and Table 2).

\section{Examination of Spread at the Border of Breast Cancer Lesions Using the Subtraction Method}

Based on the fact that the malignant lesion was visualized, whereas the benign lesion and normal tissue tended to be erased on subtracted images, we retrospectively evaluated the extent to which it was possible to diagnose tumor spread using subtracted images from 45 histo- logically proven breast cancer patients, paying special attention to intraductal spread, multiple cancerous lesions, and daughter nodules.

In some cases, cancer spread was visualized clearly on the subtracted images. However, although accuracy was higher with subtracted images than with conventional mammography (subtracted images, $75 \%$; conventional mammography $44 \%$ ), there were many false-positive cases diagnosed by subtracted images. Therefore, it was assumed that subtraction is not yet sufficient for widespread clinical use and required further study.

In case 4 (Fig 5), a 42-year-old woman was diagnosed with breast cancer. The mammogram on the left showed that breast cancer could be diagnosed by the irregular tumor shadow, but it was not possible to evaluate whether the cancer had spread around the main tumor. However, we assumed that the cancer had not spread. On the contrary, the two subtracted images on the right indicated dense shadows remaining in the

Table 2. Subtraction of Benign Diseases

\begin{tabular}{|c|c|c|c|c|c|}
\hline & \multicolumn{5}{|c|}{ Disease (no. of cases) } \\
\hline & \multicolumn{2}{|c|}{ Fibrocystic disease (8) } & Cystic disease (3) & \multicolumn{2}{|c|}{ Fibroadenoma (4) } \\
\hline $\begin{array}{l}\text { Mammography } \\
\text { observation }\end{array}$ & $\begin{array}{l}\text { Tumor shadow } \\
\text { high-density } \\
\text { area (6) }\end{array}$ & $\begin{array}{l}\text { Spicule observa- } \\
\text { tion (2) }\end{array}$ & Tumor shadow $(3)$ & \multicolumn{2}{|c|}{ Tumor shadow $\{4\}$} \\
\hline $\begin{array}{l}\text { Subtraction obser } \\
\text { vation }\end{array}$ & $\begin{array}{l}\text { Tumor shadow } \\
\text { high-density } \\
\text { area erased (6) }\end{array}$ & Spicule erased (2) & $\begin{array}{c}\text { Tumor shadow } \\
\text { erased (3) }\end{array}$ & $\begin{array}{c}\text { Tumor shadow } \\
\text { erased (2) }\end{array}$ & $\begin{array}{l}\text { Tumor shadow } \\
\text { remained (2) }\end{array}$ \\
\hline
\end{tabular}


Fig 5. Although the mammogram showed no spread other than the main tumor, subtraction recognized a dark region other than the main tumor, allowing the spread of cancer to be diagnosed. Note that mammogram $A, B$, and $C$ are for subtraction.
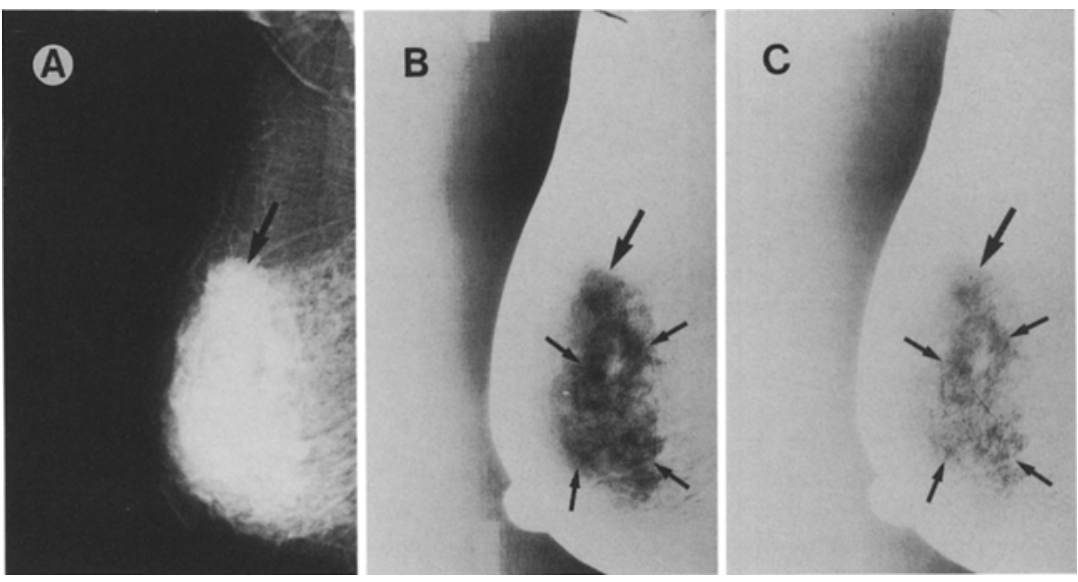

addition, abnormal lesions seen on the mammograms in patients with fibrocystic disease tended to be erased, providing useful information for clinical practice. However, there were still several cases in which the subtraction method lacked sufficient diagnostic accuracy. Two technical problems with subtraction are thought to remain. First, it is necessary to avoid misregistration stemming from motion artifacts. To overcome this problem, we will perform singleexposure dual-energy subtraction using two imaging plates. Second, we must establish the optimal conditions for subtraction. We will undertake further investigation. 